\section{Ausschreibung Oskar-Medizinpreis 2016}

Die Stiftung Oskar-Helene-Heim fördert die Wissenschaft und Forschung auf dem Gebiet der Medizin. Als besonderes Förderprojekt verleiht die Stiftung jährlich den mit $50.000 €$ dotierten Oskar-Medizinpreis. Mit diesem Medizinpreis werden hervorragende Leistungen gewürdigt und die Weiterführung von Forschungen unterstützt.

\section{Im Jahr 2016 wird dieser Medizinpreis auf dem Gebiet der}

\section{Pneumologie}

ausgeschrieben. Die pulmonale Hypertonie - auch Lungenhochdruck genannt - ist eine pathophysiologische Störung, die als Komplikation bei der Mehrzahl respiratorischer und kardiovaskulärer Krankheiten auftreten kann. Die Bezeichnung dient als Oberbegriff für verschiedenste Krankheitsbilder, bei denen der Blutdruck im Lungenkreislauf erhöht ist. Es handelt sich um eine meist chronische Erkrankung, die im fortgeschrittenen Stadium zu Rechtsherzversagen führen und damit lebensbedrohlich sein kann.

Voraussetzung für eine erfolgreiche Behandlung ist eine möglichst frühzeitige Diagnose. Da die Symptome bei der pulmonalen Hypertonie anfangs nicht spezifisch sind, kann dies zu einer deutlichen Verzögerung der richtigen Diagnosestellung und der gebotenen Therapie führen. Zur Verbesserung von Diagnostik und Therapie der verschiedenen Formen der pulmonalen Hypertonie soll mit dem Oskar-Medizinpreis 2016 ein/e Mediziner/in oder Wissenschaftler/in ausgezeichnet werden, die/der zum Thema:

\section{"Pulmonale Hypertonie"}

besonders relevante Ergebnisse der Grundlagen- und/oder klinischen Forschung in Deutschland erzielt hat. Fachübergreifende Arbeitsgruppen sind ebenfalls zur Bewerbung zugelassen.
Der prämierte Erkenntnisgewinn soll für eine breite Öffentlichkeit vermittelbar sein.

Dem Antrag sollen der Lebenslauf, die wichtigsten Arbeiten aus den letzten drei Jahren zum Thema „Pulmonale Hypertonie“ (Arbeiten, die bereits eine anderweitige Prämierung erhalten haben, sind ausgeschlossen) sowie eine inhaltliche Zusammenfassung der Forschungsergebnisse beigefügt werden, ergänzt um die weitere Forschungsplanung.

Das Preisgeld ist für Forschungszwecke nach der freien Entscheidung des Preisträgers zu verwenden. Der Preisträger hat der Stiftung diese Verwendung in geeigneter Weise zu belegen.

Der Antrag ist bis zum 31. Mai 2016 einzureichen bei der Stiftung OskarHelene-Heim, Walterhöferstr. 11, 14165 Berlin (www.stiftung-ohh.de).

Weitere Informationen erteilt der Geschäftsführer der Stiftung, Werner Ukas: werner.ukas@stiftung-ohh.de, Tel.030/8102-1100.

Zweck der Stiftung Oskar-Helene-Heim ist die Förderung von Wissenschaft und Forschung in der Medizin, insbesondere der Orthopädie, der Lungenheilkunde, Gastroenterologie und Viszeralchirurgie sowie der Orthopädietechnik.

Zu diesem Zweck unterstützt die Stiftung Forschungsprojekte oder sonstige gemeinnützige gesundheitsfördernde Vorhaben, verleiht Stipendien und vergibt jährlich den Oskar-Medizinpreis und die HeleneMedaille.

Stiftung Oskar-Helene-Heim, www.stiftung-ohh.de

\title{
Endometriose
}

\section{Herz in Gefahr!}

Die Endometriose ist eine chronisch entzündliche Erkrankung, was sich in einer hohen Konzentration an Entzündungsfaktoren und Markern für oxidativen Stress in Blut und Peritonealflüssigkeit widerspiegelt. Meist sind auch die LDL-Spiegel der Patientinnen zu hoch und die HDL-Spiegel zu niedrig. Alles Faktoren, die das Risiko für KHK erhöhen.

Ob mit der Endometriose tatsächlich das KHK-Risiko steigt, haben der Mediziner Fan $\mathrm{Mu}$ vom Brigham and Women's Hospital in Boston und seine Kollegen prospektiv an 116.430 Frauen aus der Nurses' Health Study II (1989-2009) überprüft. Sie haben verglichen, wie vie- le Frauen mit Endometriose und wie viele Frauen ohne Endometriose innerhalb des 20-jährigen Beobachtungszeitraums an einer KHK erkrankt sind. Insgesamt waren es 1438 Teilnehmerinnen, die entweder einen Myokardinfarkt erlitten oder bei denen eine Angina pectoris bestätigt oder eine Bypassoperation bzw. eine koronare Angioplastie durchgeführt bzw. ein Stent eingesetzt wurde. Frauen mit Endometriose hatten dabei ein deutlich höheres Erkrankungsrisiko.

Das Herzinfarktrisiko (RR 1,63), das Risiko für Bypass/Angioplastie/Stent (RR 1,49) wie auch das Risiko für kombinierte KHK-Endpunkte (RR 1,62) waren um etwa $50 \%$ höher als bei Frauen ohne Endometriose. Das Risiko für eine Angina stieg sogar auf das Doppelte (RR
2,07). Besonders deutlich war der Effekt bei jungen Endometriosepatientinnen bis 40 Jahre (RR kombinierter KHKEndpunkte 3,08). Danach sank das relative Risiko wieder, und ab 55 Jahren machte es keinen Unterschied mehr, ob eine Endometriose vorlag oder nicht (RR 0,98). Hysterektomie oder Oophorektomie schlugen sich ebenfalls im KHK-Risiko nieder (RR 1,51).

Für Mu und Kollegen steht somit ein Zusammenhang zwischen Endometriose und KHK fest. Betroffene Frauen seien daher über ihr KHK-Risiko aufzuklären, so die Forderungen der Forscher.

(Dagmar Kraus)

Mu Fet al. Endometriosis and Risk of Coronary Heart

Disease. Circ Cardiovasc Qual Outcomes 2016 